\title{
Usability Evaluation of the Universal Computer Workstation under Supine, Sitting and Standing Postures
}

\author{
Hsin-Chieh $\mathrm{Wu}^{1, *}$, Min-Chi Chiu ${ }^{2}$, Cheng-Lung Lee ${ }^{1}$, and Ming-Yao Bai ${ }^{1}$ \\ ${ }^{1}$ Department of Industrial Engineering and Management, Chaoyang University of Technology, \\ Taichung, Taiwan, R.O.C. \\ hcwulecyut. edu.tw \\ ${ }^{2}$ School of Occupational Therapy, Chung Shan Medical University, \\ Taichung, Taiwan, R.O.C.
}

\begin{abstract}
The purpose of this study was to evaluate the usability of the selfmade universal computer workstation. The 9 handicapped and 10 healthy adults were recruited to participate in this study, in order to understand the performances of computer operation, ratings in comfort and satisfaction for using the tested workstation in different positions. This workstation can be successfully adjusted for standing, sitting, and supine postures. This workstation also allows easy access of wheelchair. No significant differences in performances were found among supine, sitting, and standing postures. All of the participants considered this workstation comfortable. Most handicapped participants preferred to adopt supine posture to use the computer. The experimental results revealed that supine posture lead to more comfort in the lower back without decreasing performances while using a computer. Further, the healthy participants had the mean rating in satisfaction of 3.7, which was similar to that of the handicapped. It indicates that the tested workstation satisfied both the handicapped and the healthy participants. The findings of this study can provide helpful information for further improvement of a universal computer workstation.
\end{abstract}

Keywords: universal design, workstation, usability, ergonomic design.

\section{Introduction}

The computer technology has greatly advanced in the world. No matter in life or work, the use of visual display terminals (VDTs) has been very popular and indispensable. Generally, the computer workstation is designed for sitting posture. Unfortunately, for the person with chronic low back pain (LBP), seated posture also has its share of risks. In fact, the traditional VDT workstations not only hinder the use by some people with chronic LBP but have been shown to cause LBP after prolonged use [1]. Some people with chronic LBP are able to relieve their discomfort by lying in

\footnotetext{
* Corresponding author.
} 
a supine or significantly reclined posture [2]. Alternative computer workstations have been designed to allow the VDT operator to access the computer from a significantly reclined or supine posture. These postures have been shown to relieve some forms of LBP [2].

Previous study found that lying supine can reduce the intradiscal pressure as much as $75 \%$ as compared to standing upright. Whereas sitting upright increases intradiscal pressure as much as $40 \%$ when compared to standing upright [3]. To adopt a supine posture not only can reduce the lumbar intradiscal pressure but also allow people who have difficulty in standing or sitting to use a computer. Although lying supine has the advantage of more comfort in lower back, someone may concern about that the operation performance may be decreased by the supine posture. Studies are needed to investigate whether or not body posture affects computer operation performance. Therefore, a universal computer workstation was made here for people to adopt different postures such as supine, sitting, and standing postures when using a computer.

The main purpose of this study was to investigate the effect of body posture on the performance and subjective comfort while using a computer. The handicapped and healthy adults were recruited to participate in this study, in order to understand whether these two groups lead to different results while using this universal computer workstation. We expected that this workstation can actually solve the problems of the handicapped and people with chronic LBP. Further, the users' recommendations this workstation were also collected, in order to improve the self-made universal computer workstation.

\section{Methods}

\subsection{Subjects}

Nineteen people, including ten healthy adults and nine young handicapped persons, participated in this study. The healthy adults all have no musculoskeletal disorders. The young handicapped persons have lower limb disabilities or spinal injuries, and need to rely on a wheelchair or crutches or other aids; but their mental abilities and hands are able to operate a computer. All of the subjects weekly use a computer for more than 10 hours. The entire participants are all right-handed, and must sign the written consent of the test.

\section{$2.2 \quad$ Equipments}

The self-made universal computer workstation was used here. This workstation had a base of electric lifting table, and it was designed to be adjusted for standing, sitting, and supine postures (as shown in Fig. 1). The screen can be adjusted from 0 to 90 degrees forward; keyboard adjustment range is from 0 to 50 degrees from the desktop plain. 


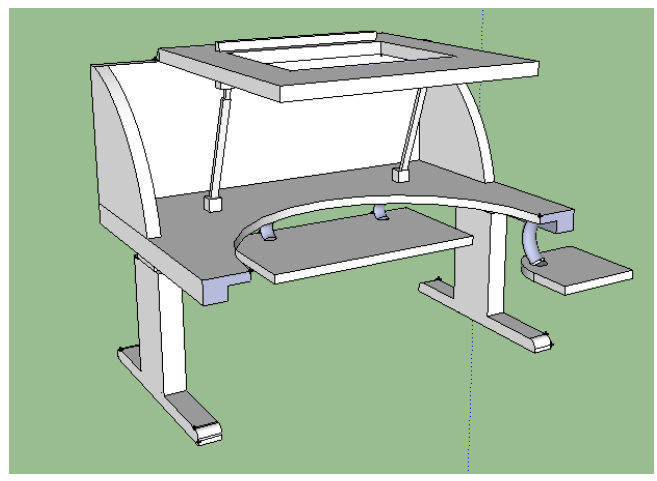

(a) Supine

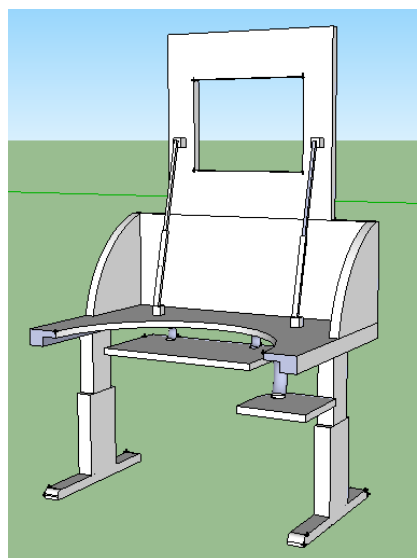

(b) Sitting

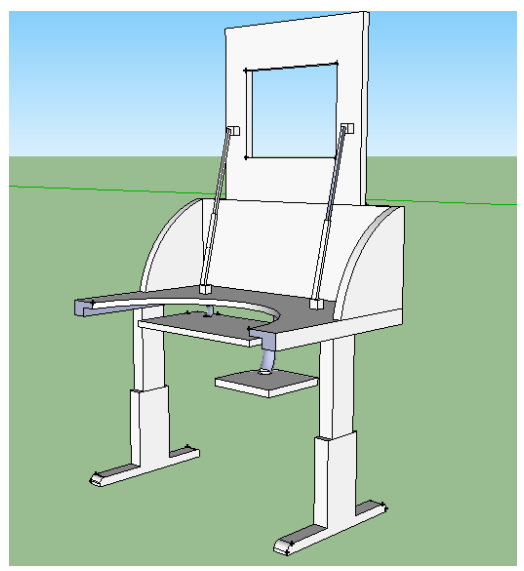

(c) Standing

Fig. 1. Illustrations of (a) supine, (b) sitting, and (c) standing positions for using the tested workstation

\subsection{Usability Test Protocol}

Computer Operations. Subjects should type Chinese words in accordance with the articles shown on the computer display, and the article input time was ten minutes. After the Chinese typing test, the score and error rate were calculated as typing performances.

After the typing test, the mouse drag-and-drop test was required to perform by subjects. Each subject was required to select twelve objects evenly distributed on a circle surrounding a central box, and then drag each object separately into the box (Fig. 2). The program automatically record the completion time until all objects were moved to the central box. This test repeated three times, and the average completion time was the performance of the mouse operation. 


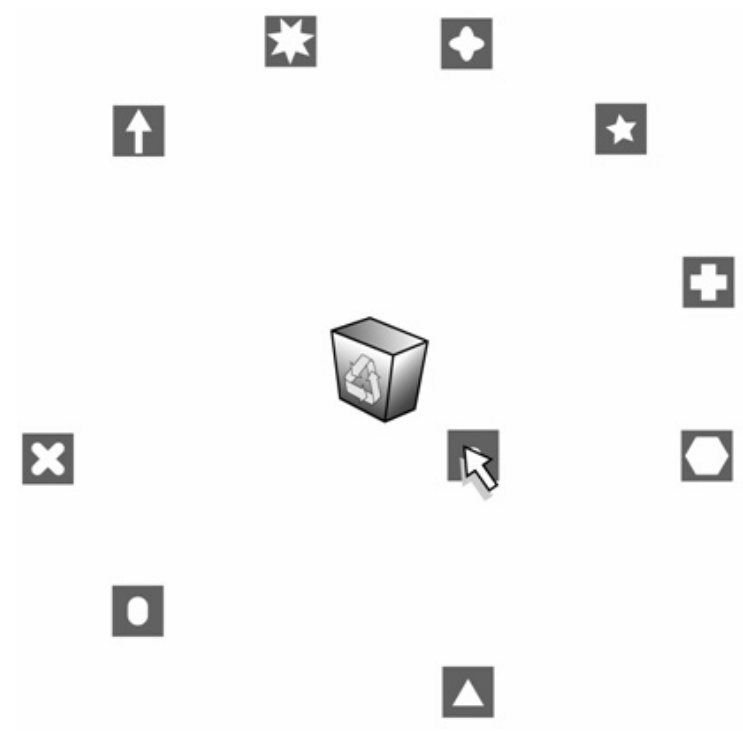

Fig. 2. An Illustration of mouse drag-and-drop test

Subjective Evaluations. The subjective evaluation included: (1) subjective ratings in comfort and (2) satisfaction ratings. Subjective ratings in comfort had a seven-point Likert scale, 1 representing 'very uncomfortable' and 7 representing 'very comfortable'. Eight body parts, including eyes, neck, shoulder, upper arm, forearm, wrist, upper back and lower back, were designed in the subjective comfort questionnaire. These data were collected after the use of the tested workstation.

In addition, subjects were asked to rate satisfaction of the tested workstation along eight items:

1. Workstations can be adjusted for standing, supine or sitting positions.

2. Various computer components can be easily placed in this workstation.

3. Can easily enter the workstation.

4. The tilt angle of the screen and viewing distance can be adjusted according to your needs.

5. Mouse and keyboard positions and tilt angle can be adjusted according to your needs.

6. Using the computer, operating the mouse and keyboard comfortably.

7. Can easily leave the workstation.

8. Can easily remove various computer components.

Each item had a five-point Likert scale, with 1 representing 'very unsatisfactory' and 5 representing 'very satisfactory'. 


\subsection{Experimental Settings}

In order to reduce the interference of other variables, this study tried to control the following factors:

- The computer jobs, screen brightness and contrast were the same. The ambient illumination was controlled at $500 \sim 600$ lux.

- The subjects were in good mental condition during the experiment. The input method taken by the participants was Microsoft New Phonetic, and the experimental room had no noise, so as not to affect the degree of concentration of the participants.

\section{$3 \quad$ Results}

\subsection{Operation Performances}

Table 1 shows the computer operation performances for the healthy adults and the young handicapped subjects in the standing, sitting, and supine positions. No data were available for the young handicapped subjects in the standing position because they were not able to adopt standing posture. The ANOVA results indicated that body posture had little effect $(\mathrm{p}>0.05)$ on typing score, error rate, and mouse drag time. Further, it is obvious that the young handicapped subjects had worse operation performances than the healthy adults.

\subsection{Subjective Evaluation Results}

Table 2 shows the descriptive statistics (mean \pm SD) of subjective comfort ratings in eight body parts for the three different positions. No data were available for the young handicapped subjects in the standing position because they were not able to adopt standing posture. The ANOVA results indicated that body posture had a significant effect $(\mathrm{p}<0.05)$ on the comfort ratings in lower back. Post hoc comparisons were then performed by Turkey method. The analyzed results revealed that supine posture had significantly better comfort ratings in lower back compared with standing and sitting postures. Further, it is obvious that the young handicapped subjects had similar comfort ratings as compared to those of the healthy adults.

Finally, the results of satisfaction ratings show that the healthy adults had the mean ratings in satisfaction of 3.7, and the handicapped also had the mean ratings in satisfaction of 3.7. It indicates that this workstation satisfied both the handicapped and the healthy participants.

Table 1. Computer operation performances in the standing, sitting, and supine positions

\begin{tabular}{lcccccc}
\hline & \multicolumn{3}{c}{ The healthy adults } & \multicolumn{3}{c}{ The young handicapped } \\
Performance & Standing & Sitting & Supine & Standing & Sitting & Supine \\
\hline Typing score & $516 \pm 168$ & $500 \pm 169$ & $435 \pm 141$ & N/A & $51 \pm 77$ & $44.4 \pm 74.6$ \\
Error rate (\%) & $5.3 \pm 10.4$ & $1.6 \pm 1.6$ & $2.2 \pm 2.8$ & N/A & $18.5 \pm 18.2$ & $32.4 \pm 37.5$ \\
Drag time (s) & $13.9 \pm 1.4$ & $14.1 \pm 2.0$ & $14.9 \pm 1.8$ & N/A & $58.9 \pm 22.4$ & $52.1 \pm 15.7$ \\
\hline
\end{tabular}


Table 2. Subjective comfort ratings for the standing, sitting, and supine postures

\begin{tabular}{ccccccc}
\hline & \multicolumn{3}{c}{ The healthy adults } & \multicolumn{3}{c}{ The young handicapped } \\
Body part & Standing & Sitting & Supine & Standing & Sitting & Supine \\
\hline Eyes & $4.3 \pm 1.1$ & $4.5 \pm 1.0$ & $4.5 \pm 1.5$ & N/A & $4.7 \pm 1.1$ & $4.1 \pm 0.6$ \\
Neck & $4.2 \pm 1.0$ & $4.5 \pm 1.0$ & $5.1 \pm 1.6$ & N/A & $4.8 \pm 1.4$ & $4.3 \pm 1.4$ \\
Shoulder & $4.2 \pm 1.2$ & $4.0 \pm 1.1$ & $4.8 \pm 1.8$ & N/A & $4.3 \pm 1.1$ & $4.6 \pm 1.3$ \\
Upper arm & $3.8 \pm 1.0$ & $3.9 \pm 0.7$ & $4.4 \pm 1.7$ & N/A & $4.3 \pm 1.1$ & $4.8 \pm 1.2$ \\
Forearm & $3.7 \pm 1.1$ & $3.1 \pm 0.9$ & $3.5 \pm 1.6$ & N/A & $4.1 \pm 1.3$ & $4.6 \pm 1.4$ \\
Wrist & $3.7 \pm 1.3$ & $3.2 \pm 1.0$ & $3.1 \pm 1.4$ & N/A & $3.2 \pm 1.0$ & $3.8 \pm 1.4$ \\
Upper back & $4.1 \pm 1.4$ & $4.4 \pm 0.8$ & $5.4 \pm 1.4$ & N/A & $5.0 \pm 1.2$ & $4.9 \pm 1.4$ \\
Lower back* & $4.0 \pm 1.3$ & $4.5 \pm 0.9$ & $5.6 \pm 1.3$ & N/A & $4.6 \pm 1.6$ & $5.1 \pm 1.1$ \\
\hline
\end{tabular}

PS: 1 representing 'very uncomfortable' and 7 representing 'very comfortable'.

* Body posture had a significant effect $(\mathrm{p}<0.05)$ on the comfort ratings in lower back.

\section{Discussion}

The main purpose of this study was to evaluate the usability of the self-made universal computer workstation in three different body postures. Computer operation performances, subjective comfort and satisfaction were collected. Experimental results show that the participants had similar computer operation performances among standing, sitting, and supine postures. It implies that the tested workstation could be adequately adjusted for operating a computer in standing, sitting, and supine positions and inconsequently caused similar performances at different body postures. In addition, the comfort rating results show that supine posture had significantly better comfort ratings in lower back than those of standing and sitting postures. Therefore, it should be noted that supine posture can improve lower back comfort without decreasing operation performances while using a computer. However, the operation time of each trial was only 30 minutes in the current study. More studies are required to investigate whether the current findings can be applied in the prolonged VDT operation tasks.

Acknowledgments. The authors thank the National Science Council of the Republic of China for financially supporting this research (Contracts No. NSC98-2221-E-324013 and NSC99-2628-E-324-024).

\section{References}

1. Bendix, T.: Low back pain and seating. In: Lueder, R., Noro, K. (eds.) Hard Facts about Soft Machines- The Ergonomics of Seating, pp. 147-155. Taylor \& Francis Ltd., London (1994)

2. Haynes, S., Williams, K.: Product review of alternative computer workstations as possible workplace accommodations for people with chronic low back pain. Technology and Disability $19,41-52$ (2007)

3. Nachemson, A.: The lumbar spine, an orthopedic challenge. Spine 1(1), 59-69 (1976) 